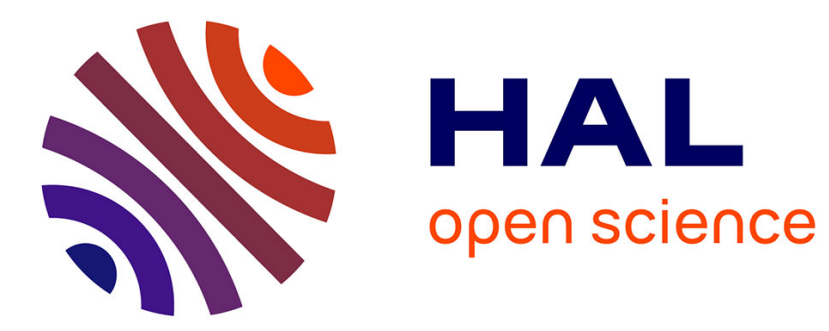

\title{
Nutrition anti-inflammatoire et MICI : que dire à nos patients?
}

Xavier Hébuterne

\section{To cite this version:}

Xavier Hébuterne. Nutrition anti-inflammatoire et MICI : que dire à nos patients?. Nutrition Clinique et Métabolisme, 2019, 33, pp.126 - 130. 10.1016/j.nupar.2019.02.003 . hal-03484753

\section{HAL Id: hal-03484753 \\ https://hal.science/hal-03484753}

Submitted on 20 Dec 2021

HAL is a multi-disciplinary open access archive for the deposit and dissemination of scientific research documents, whether they are published or not. The documents may come from teaching and research institutions in France or abroad, or from public or private research centers.
L'archive ouverte pluridisciplinaire HAL, est destinée au dépôt et à la diffusion de documents scientifiques de niveau recherche, publiés ou non, émanant des établissements d'enseignement et de recherche français ou étrangers, des laboratoires publics ou privés.

\section{다)(1) $(5$}

Distributed under a Creative Commons Attribution - NonCommerciall 4.0 International 


\title{
Nutrition anti-inflammatoire et $\mathrm{MICl}$ : que dire à nos patients ?
}

\author{
Pr Xavier Hébuterne, \\ Service de gastro-entérologie et Nutrition Clinique, \\ CHU de Nice, France.
}

Correspondance : Pr. Xavier Hébuterne, Gastro-entérologie et Nutrition Clinique, Hôpital de l'Archet, 151 Route Saint Antoine de Ginestière, 06202 Nice cedex 03 France.

Téléphone : (33) 492036168. Fax : (33) 492036575.

e-mail: hebuterne.x@chu-nice.fr 


\section{Résumé}

- Une alimentation riche en fruits et légumes, en poisson et réduite en viande rouge et sucreries est associée à un risque moindre de développer une $\mathrm{MICl}$. II n'existe pas de "régime anti-inflammatoire " dont l'efficacité est démontrée. A l'inverse, ils peuvent être coûteux, ils entraînent des carences, ils réduisent le plaisir de manger et sont désocialisants. Au cours des $\mathrm{MICl}$, la prise prolongée de compléments alimentaires n'est pas recommandée : elle est en général inefficace et peut être dangereuse. Il faut privilégier une alimentation plaisir, saine, variée, équilibrée et adaptée aux symptômes de la maladie. L'accompagnement par un(e) diététicien(e) est recommandé.

\section{Abstract}

- A diet rich in fruits and vegetables, fish, and lower in meat and sweets is associated with a lower risk of developing IBD. There is no "anti-inflammatory diet" whose effectiveness is demonstrated. These regimens can be expensive, they cause deficiencies, and they reduce the pleasure of eating and are desocializing. During IBD, prolonged intake of oral supplements is not recommended: it is usually ineffective and possibly dangerous. Doctors must promote a pleasure diet, healthy, varied, balanced and adapted to the symptoms of the disease. Accompaniment by a dietician is recommended.

Mots-clés : maladie inflammatoire de l'intestin, maladie de Crohn, rectocolite hémorragique, nutrition, régime, compléments alimentaires

Key words: inflammatory bowel disease, Crohn's disease, ulcerative colitis, nutrition, regimen, oral supplements 
L'augmentation considérable de l'incidence des $\mathrm{MICl}$, surtout dans les pays en voie d'industrialisation, est expliquée par l'existence de facteurs environnementaux qui favorisent la survenue d'une $\mathrm{MICl}$ sur un terrain génétiquement prédisposé (1). Parmi ces facteurs environnementaux, l'alimentation est au premier plan.

\section{Alimentation et risque de survenue d'une $\mathrm{MICl}$}

II est logique de penser que les $\mathrm{MICl}$ sont en partie liées à ce que nous mangeons et des études épidémiologiques ont montré qu'une alimentation riche en fruits et légumes et en acides gras n-3 provenant des poissons est associée à un risque plus faible de $\mathrm{MICl}(2)$. A l'inverse, une alimentation plus riche en viande rouge, en sucres, boissons sucrées et confiseries et une consommation basse en légumes et légumineuses augmentent le risque de $\operatorname{MICl}(3,4)$. Plus que le type d'aliment, il semble que le risque de survenue d'une $\mathrm{MICl}$ soit associé à la typologie alimentaire. Ainsi, dans l'étude de Racine et al. (3) le risque de développer une $\mathrm{RCH}$ augmentait chez les personnes qui avaient une consommation élevée en sucres, boissons sucrées et confiseries associée à une consommation basse en légumes et légumineuses; à l'inverse, il n'augmentait pas chez les sujets dont la consommation élevée en sucres, boissons sucrées et confiseries, était associée à une consommation élevée en légumes, légumineuses et en fruits. L'étude d'Ananthakrishnan et al. (4) suggère que la typologie alimentaire de l'adolescence influence le risque de développer une $\mathrm{MICl}$ plus tard. En 1997, 40,5\% des 116.686 femmes qui participent à la Nurses' Health Study ont renvoyé un questionnaire détaillé concernant leur alimentation à l'âge de 13 à 17 ans. Elles étaient âgées de 35 à 51 ans et indemnes de MICl. En 2011 (763.229 personnes/année), 70 cas incidents de $\mathrm{MC}$ et 103 de $\mathrm{RCH}$ ont été déclarés. Le risque de développer une $\mathrm{MICl}$ était significativement réduit dans le groupe de femmes qui avaient eu l'alimentation la plus prudente, $c^{\prime}$ est à dire la plus riche en fruits et légumes et en poisson (pour le $4^{\text {ème }}$ quartile : $H R=0,47 ;$ IC 95\% : 0,23-0,98, P = 0,04) (Figure 1).

A côté des aliments, le rôle d'éventuels contaminants de l'alimentation a été évoqué. Les additifs alimentaires comme les maltodextrines, les carraghénanes (algues) et la carboxyméthyle cellulose sont suspectés. Ces agents sont largement utilisés dans l'industrie alimentaire comme épaississant ou agent de remplissage pour augmenter le volume d'aliments transformés, comme des desserts instantanés et les gélatines, les sauces et les vinaigrettes, les produits de boulangerie, les croustilles, viandes séchées, yogourts, barres 
nutritives, shakes de substituts de repas (5). Un remarquable travail expérimental suggère que l'addition de toutes petites quantités de carboxymethylcellulose ou de polysorbate-80 dans l'eau de boisson de souris entraîne la survenue de colites expérimentales et modifie profondément le microbiote de ces animaux (6). S'il n'existe pas à ce jour de données épidémiologiques qui suggèrent que les additifs alimentaires sont associés à un risque accru de $\mathrm{MICl}$, c'est dû essentiellement au fait que les questionnaires utilisés dans les grandes cohortes (Nurses' health study, EPIC, ...) n'étaient pas formatés pour répondre à cette question. Une étude récente provenant de la cohorte Nutrinet Santé montre qu'une augmentation de $10 \%$ de la proportion d'aliments ultra-transformés dans l'alimentation est associé à une augmentation du risque de cancer de $10 \%$ (7). On attend avec impatience les résultats sur les $\mathrm{MICl}$ provenant de cette cohorte.

\section{Faut-il suivre un régime lorsque l’on a une $\mathrm{MICl}$ et si oui lequel?}

Chez un malade atteint de $\mathrm{MICl}$, en dehors de quelques situations particulières (sténose intestinale par exemple), la survenue d'une poussée n'est pas liée directement à l'alimentation.

Lorsqu'il existe une poussée inflammatoire sévère, on peut conseiller au patient de suivre un régime sans résidu pendant quelques jours afin de réduire le nombre de selles et les douleurs abdominales. L'objectif est de diminuer le volume des selles et leur nombre durant la phase de poussée. II repose sur la suppression des aliments contenant des fibres tels que les fruits et les légumes qu'ils soient crus ou cuits ainsi que les céréales complètes et, de façon plus stricte, des produits laitiers si leur consommation amplifie les symptômes. Dès que la situation s'améliore, il faut les réintroduire progressivement. A ce stade, une consultation avec un(e) diététicien(ne) spécialisé(e) est recommandée afin d'aider le patient à adapter son alimentation à ses difficultés digestives tout en conservant un bon équilibre alimentaire. Dans certains cas l'exclusion totale de l'alimentation et la nutrition artificielle notamment la nutrition entérale chez l'enfant (8) et en cas de complication (sténose, abcès), permet d'obtenir la rémission de la maladie (2).

Lorsque la maladie est en rémission, il n'y a pas de régime type capable de prévenir une poussée. Ceci ne veut pas pour autant dire que le patient peut manger n'importe quoi. L'alimentation doit être la plus variée possible, elle doit respecter les grands équilibres alimentaires et doit être suffisamment riche en énergie et protéines pour couvrir les besoins 
et ainsi maintenir un bon état nutritionnel. La consommation de fruits et de légumes est recommandée de manière à couvrir les besoins en vitamines, micronutriments et antioxydants. Il est par ailleurs essentiel et tout à fait possible que le plaisir de l'alimentation soit conservé. Bien entendu, l'alimentation doit être adaptée à la tolérance digestive du patient qui varie en fonction du niveau d'activité de la maladie. Tout malade diagnostiqué $\mathrm{MICl}$ devrait avoir, dès l'annonce du diagnostic de sa maladie, une information personnalisée concernant son alimentation. II devrait également avoir un accompagnement diététique et nutritionnel au cours du suivi, c'est le moins pour une maladie qui interagit autant avec son alimentation (2).

Les principales erreurs alimentaires que l'on rencontre chez les malades sont la prolongation d'un régime sans résidu après une poussée de la maladie; l'élimination systématique d'aliments tels que le gluten, le lactose, les fruits et légumes, voire la viande, dans l'espoir de prévenir une poussée ; le régime sans sel strict au cours d'une corticothérapie alors qu'il aurait été préférable d’augmenter les protéines et de réduire les graisses ; la prise de compléments alimentaires au long cours sans avis médical, etc... Mais un fléau guette les malades : ce sont les régimes dit anti-inflammatoires ou anti-MICl. Comment ne pas comprendre un patient souvent désemparé qui, cherchant une solution à son problème et tombant sur des sites ou des ouvrages qui affirment de manière péremptoire que la solution qu'ils proposent est miraculeuse, modifie son régime ou teste des aliments ou des complément alimentaires dont on lui a vanté les mérites. Citons les jus de légumes antiinflammatoires, les probiotiques magiques anti-MICI, les cures de "détox », mais surtout les sinistres régimes chasseurs-cueilleurs et paléolithiques, basés sur le concept binaire suivant : les $\mathrm{MICl}$ et d'autres maladies n'existaient pas avant, mangeons donc comme avant pour ne pas être malade ! C'est oublier que de nombreuses études scientifiquement indiscutables n'ont pas permis de montrer de bénéfice clair à un régime d'exclusion quel qu'il soit sur les poussées de $\mathrm{MICl}$.

Le jeûne thérapeutique " détoxifiant » est également très en vogue. L'idée vient du cancer et du concept qu'il faut limiter la consommation de sucres pour ne pas nourrir la tumeur. Ce questionnement peut être rapproché de l'engouement actuel pour les régimes de jeûne intermittent, de restriction calorique ou le régime cétogène. L'avidité de certaines cellules tumorales pour le glucose ne peut être contestée. Cependant, de nombreuses cellules 
normales ne peuvent se passer de sucres en situation d'agression ou de maladie inflammatoire. Les études expérimentales et les études cliniques actuellement disponibles ne permettent pas de conclure au bénéfice de ces régimes sur la toxicité des traitements anticancéreux ou sur l'évolution tumorale. Les conclusions du rapport de l'Institut National du Cancer sont claires : au cours du cancer, le jeûne thérapeutique est inefficace ; il favorise la dénutrition et la sarcopénie (9). Ces conclusions peuvent, dans l'état actuel des connaissances, être extrapolées aux maladies inflammatoires.

Le régime pauvre en FODMAPs [F = Fermentescibles; $\mathrm{O}=$ Oligosaccharides (fructanes et galacto-oligosaccharides ou GOS) ; $\mathrm{D}=$ Disaccharides (lactose) ; $\mathrm{M}=$ Monosaccharides (fructose en excès du glucose) ; $A=A$ nd ; $P=$ Polyols (sorbitol, mannitol, xylitol et maltitol)] dont l'intérêt est démontré dans le syndrome de l'intestin irritable est de plus en plus étudié au cours des $\mathrm{MICl}$. II limite les aliments contenant des glucides ou sucres que l'on dit «fermentescibles». Ces glucides sont faiblement absorbés par l'intestin grêle. Cela produit des substances qui arrivent rapidement dans le côlon et sont dégradées par le microbiote. Cette fermentation colique rapide peut provoquer des ballonnements, des gaz et des douleurs abdominales. Les aliments concernés sont par exemple la banane, le pamplemousse, les carottes, les haricots verts, les pommes de terre, le riz, l'avoine, le sarrasin, le lait sans lactose, les fromages à pâte dure, l'huile d'olive... Dans certains cas, la réduction de la quantité d'aliments riches en FODMAPs améliore les symptômes (10). Un essai contrôlé randomisé comparant un régime pauvre en FODMAPs à un régime normal chez 52 malades ayant une $\mathrm{MICl}$ en rémission (maladie quiescente selon le score PGA, un taux de calprotectine fécale $<250 \mu \mathrm{g} / \mathrm{g}$ et de CRP $<10 \mathrm{mg} / \mathrm{L}$ ) et des symptômes fonctionnels invalidants selon la classification de Rome III pour le ballonnement et la diarrhée, a été présenté à la DDW 2018. Le suivi du régime était évalué par des questionnaires quotidiens. Après quatre semaines, la proportion de malades se disant améliorés selon leur propre évaluation des symptômes était plus élevée dans le bras régime pauvre en FODMAPs. Le score total IBS-SSS (Irritable Bowel Syndrome - Symptom Severity Score) diminuait de manière plus importante dans le bras "régime" que chez les témoins, sans atteindre un seuil significatif (Figure 2). Par contre, les taux de calprotectine fécale et de CRP, ainsi que la composition du microbiote, n'étaient pas différents entre les deux groupes à la semaine 4 (11). Ce type de régime semble donc agir plus sur les symptômes que sur l'inflammation. II 
peut être utile aux patients mais le mettre en place efficacement est très complexe et peut entraîner des carences, il est nécessaire de consulter un(e) diététicien(ne) spécialisé(e) dans ce domaine.

\section{Faut-il prendre des compléments alimentaires ?}

On sait qu'une alimentation riche en huile de poisson (les acides gras oméga 3) est associée à un risque réduit de développer une $\mathrm{MICl}$. La tentation était grande de proposer une supplémentation en oméga 3 chez les malades atteints de $\mathrm{MICl}$. Quelques études ouvertes semblant montrer un bénéfice, une étude randomisée de phase 2 utilisant des capsules d'EPA à libération entérale (pour en améliorer la tolérance) chez des patients en rémission de leur MC, a été mise en place. Ses résultats étaient en faveur d'une efficacité de ce traitement (12). Malheureusement, l'étude de phase 3, réalisée chez plusieurs centaines de malades porteurs d'une MC en rémission, en Europe et aux USA, n'a pas permis de montrer de réduction des poussées chez les malades à qui on avait enrichi l'alimentation avec des huiles de poissons (13).

De très nombreux compléments alimentaires, notamment à base de plantes, sont supposés agir contre l'inflammation et sont proposés aux malades par divers moyens notamment internet. Très peu ont fait l'objet d'études démontrant leur efficacité et leur innocuité (14). La curcumine, très à la mode et prônée efficace dans de très nombreuses maladies, a fait l'objet d'études prometteuses dans la RCH (15). Un travail multicentrique récent dans la MC ne semble pas confirmer ces résultats (16). Dans une autre étude, une plante médicinale traditionnelle chinoise, le qing-dai (indigo naturalis), vient d'être testée dans la RCH. Elle semble efficace, mais près de $20 \%$ des patients ont développé des anomalies biologiques hépatiques et l'essai a été stoppé prématurément car un patient a développé une hypertension artérielle pulmonaire (17). La prise systématique et prolongée de compléments alimentaires peut être dangereuse et n'est pas recommandée.

La question de l'intérêt de la prise de probiotiques se pose également souvent. Il existe de très nombreux produits de qualité très variable. Les probiotiques actuels n'ont aucun intérêt démontré dans la MC (18). Certains probiotiques comme le VSL 3, ont montré leur intérêt dans la RCH et la pochite au cours de la poussée et en prévention des récidives (16) et peuvent être proposés. 
Concernant les vitamines et les micronutriments, seule une carence confirmée par un bilan sanguin justifie une supplémentation. Au cours des $\mathrm{MICl}$, la recherche systématique de carences en vitamines B9, B12 et D et en fer, voire en zinc chez les malades qui présentent une diarrhée importante semble justifiée. La vitamine D possède d'importantes propriétés immunomodulatrices, qui suggèrent un effet bénéfique au cours de la MC. Un taux bas de vitamine $\mathrm{D}$ est associé à un risque plus élevé de développer une maladie de Crohn. Chez les patients porteurs de $\mathrm{MICl}$, une insuffisance en vitamine $\mathrm{D}$ est fréquente et semble associée à une maladie plus active $(19,20)$.

\section{Faut-il changer son mode d'alimentation quand on déclare une $\mathrm{MICl}$ ?}

Des témoignages de patients suggèrent qu'une modification du mode d'alimentation et plus généralement de leur mode de vie, leur ont permis de mieux gérer leur maladie (21). Le concept de « healthy diet » repose sur quatre piliers :

1. Privilégier les produits frais : fruits et légumes de saison en évitant les produits industriels, les plats tout prêts trop salés et sucrés avec de nombreux additifs alimentaires.

2. Préférer le poisson (sardine, maquereau, thon, saumon, cabillaud, ...), les fruits de mer (crabe, crevettes, moules, ...) et la viande blanche, limiter la viande rouge (une à deux fois par semaine).

3. Cuisiner maison. Privilégier les cuissons à la vapeur, au four ou à la planche. Limiter le sel et les matières grasses pour la cuisson. Utiliser de l'huile d'olive.

4. Adopter le végétal : certaines protéines végétales peuvent remplacer les protéines animales (graines de courges, avoine, tofu, soja, ...).

Le mode de vie " healthy » consiste également à faire régulièrement de l'activité physique, ce qui est bénéfique sur l'inflammation, à ne pas fumer et ne boire de l'alcool que très modérément voire pas du tout.

\section{Comment le médecin peut-il conseiller son patient ?}

Le médecin doit aborder le sujet de l'alimentation dès la première consultation. II doit être proactif et ne pas attendre que son malade lui en parle et lui annonce qu'il suit tel ou tel régime découvert sur internet ou grâce aux conseils d'amis. II faut essayer d'en savoir plus sur ce que le patient mange afin de détecter les erreurs alimentaires et accompagner un 
éventuel changement des habitudes alimentaires. II faut donner des conseils de bon sens et les personnaliser. L'imprimé type au logo du service ou du cabinet avec le "régime $\mathrm{MICl}$ » n'existe pas il faut l'expliquer au malade qui bien souvent l'attend. II faut privilégier une alimentation plaisir plutôt qu'une alimentation sanction en adaptant les conseils à l'état digestif (poussée, stomie, grêle court, rémission, ...) et aux préférences du patient. II est possible de s'aider d'outils (livret, fiches, etc...). II est aussi très souvent utile d'orienter le patient vers des ateliers diététiques, une consultation diététique, un programme d'éducation thérapeutique. II faut également peser le malade à chaque consultation et détecter les carences (fer, Vit B9, Vit B12, Vit D).

\section{Conclusion}

L'alimentation est un point clé de la prise en charge des MICI. II faut conseiller le patient et le mettre en garde contre les pièges et les recettes miracles qu'il rencontrera dans son entourage, sur internet et les réseaux sociaux. Le recours systématique à un(e) diététicien(ne) et un point majeur comme le sont le développement de l'éducation thérapeutique et des ateliers diététiques réalisés par des professionnels de santé formés et compétents. Faire de l'alimentation son alliée et non son ennemie : voilà l'enjeu de la nutrition pour la personne atteinte de $\mathrm{MICl}$. 


\section{Références :}

1. Ng SC, Shi HY, Hamidi N, et al. Worldwide incidence and prevalence of inflammatory bowel disease in the 21st century: a systematic review of population-based studies. Lancet. 2018 Dec 23;390:2769-78.

2. Forbes $A$, Escher J, Hébuterne $X$, et al. ESPEN guideline: Clinical nutrition in inflammatory bowel disease. Clin Nutr. 2017;36:321-7

3. Racine A, Carbonnel F, Chan SS, et al. Dietary Patterns and Risk of Inflammatory Bowel Disease in Europe: Results from the EPIC Study. Inflamm Bowel Dis. 2016 Feb;22:345-54

4. Ananthakrishnan AN, Khalili $H$, Song $M$, et al. High School Diet and Risk of Crohn's Disease and Ulcerative Colitis. Inflamm Bowel Dis. 2015;21:2311-9.

5. Ruemmele FM. Role of Diet in Inflammatory Bowel Disease. Ann Nutr Metab. 2016;68 Suppl 1:33-41.

6. Chassaing B, Koren $\mathrm{O}$, Goodrich JK, et al. Dietary emulsifiers impact the mouse gut microbiota promoting colitis and metabolic syndrome. Nature. 2015;519:92-6. Lang A, Salomon N, Wu JC, et al.

7. Fiolet T, Srour B, Sellem L, et al. Consumption of ultra-processed foods and cancer risk: results from NutriNet-Santé prospective cohort. BMJ. 2018;360:k322.

8. Swaminath A, Feathers A, Ananthakrishnan AN, Falzon L, Li Ferry S. Systematic review with meta-analysis: enteral nutrition therapy for the induction of remission in paediatric Crohn's disease. Aliment Pharmacol Ther. 2017;46:645-56.

9. Rapport du Réseau NACRe " Jeûne, régimes restrictifs et cancer : revue systématique des données scientifiques et analyse socio-anthropologique sur la place du jeûne en France ". Novembre 2017. https://www6.inra.fr/nacre/Le-reseau-NACRe/Publications/RapportNACRe-jeune-regimes-restrictifs-cancer-2017.

10. Gibson PR. Use of the low-FODMAP diet in inflammatory bowel disease. J Gastroenterol Hepatol. 2017;32:40-42.

11. Cox SR. Low FODMAP diet improves functional-like gastrointestinal symptoms but Reduces bifidobacteria and faecalibacterium prausnitzii in quiescent Inflammatory bowel disease: a randomised controlled trial and Metagenomic analysis. DDW 2018 ; abstract 902.

12. Belluzzi A, Brignola C, Campieri M, Pera A, Boschi S, Miglioli M. Effect of an enteric-coated fish-oil preparation on relapses in Crohn's disease. N Engl J Med. 1996 Jun 13;334:1557-60

13. Feagan BG, Sandborn WJ, Mittmann U, et al. Omega-3 free fatty acids for the maintenance of remission in Crohn disease: the EPIC Randomized Controlled Trials. JAMA. 2008;299:1690-7.

14. Ng SC, Lam YT, Tsoi KK, Chan FK, Sung JJ, Wu JC. Systematic review: the efficacy of herbal therapy in inflammatory bowel disease. Aliment Pharmacol Ther. 2013;38:854-63

15. Lang A, Salomon N, Wu JC, et al. Curcumin in Combination With Mesalamine Induces Remission in Patients With Mild-to-Moderate Ulcerative Colitis in a Randomized Controlled Trial. Clin Gastroenterol Hepatol. 2015;13:1444-9

16. Buisson et al. En préparation

17. Naganuma M, Sugimoto S, Mitsuyama K, et al. Efficacy of Indigo Naturalis in a Multicenter Randomized Controlled Trial of Patients With Ulcerative Colitis. Gastroenterology. 2018;154:935-47.

18. Derwa Y, Gracie DJ, Hamlin PJ, Ford AC. Systematic review with meta-analysis: the efficacy of probiotics in inflammatory bowel disease. Aliment Pharmacol Ther. 2017;46:389-400.

19. Ananthakrishnan AN, Khalili H, Higuchi LM, et al. Higher predicted vitamin D status is associated with reduced risk of Crohn's disease. Gastroenterology. 2012;142:482-9.

20. Ananthakrishnan AN, Cagan A, Gainer VS, et al. Normalization of plasma 25-hydroxy vitamin $D$ is associated with reduced risk of surgery in Crohn's disease. Inflamm Bowel Dis. 2013;19:1921-7.

21. Deumier J. Diagnostiquée Crohn. Changer d'alimentation m'a changé la vie. Eds Flammarion 2017. 


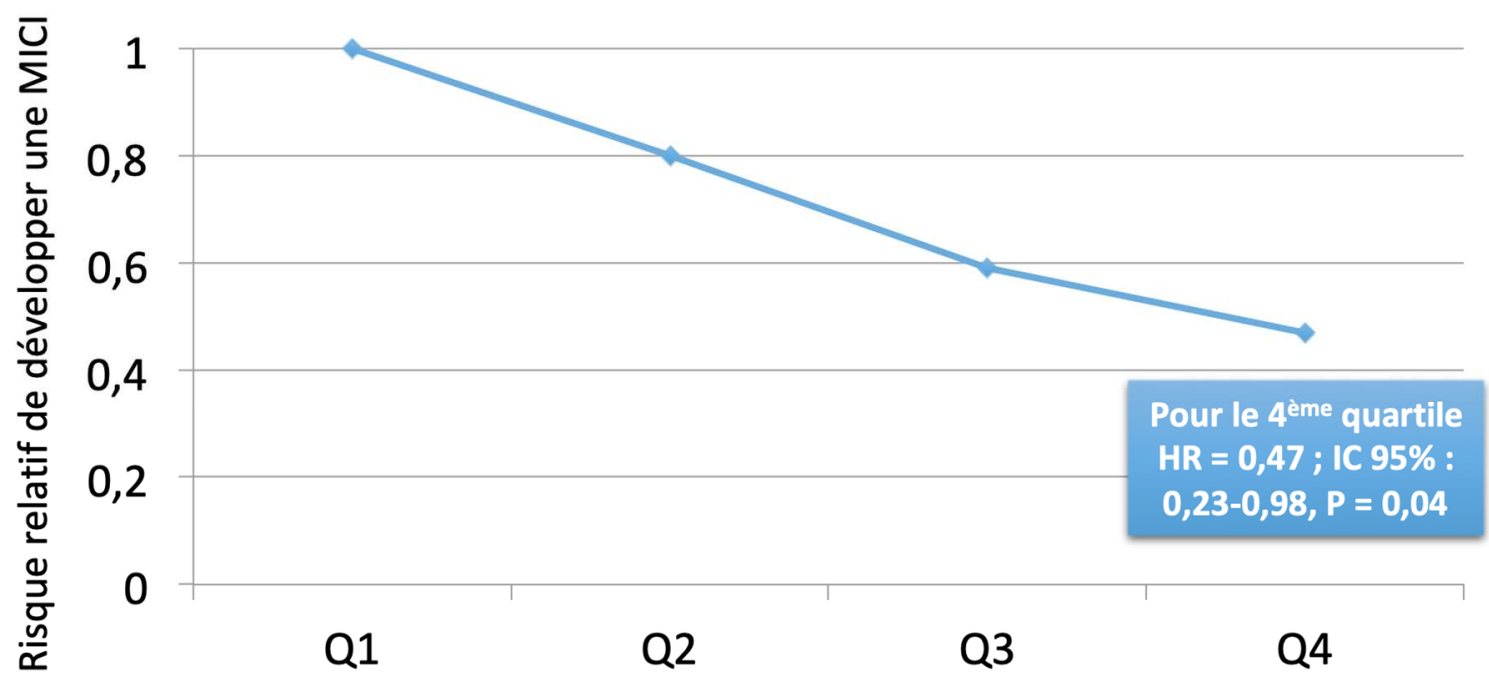

Alimentation prudente (riche en fruits et légumes et en poisson)

Figure 1: risque de développer une $\mathrm{MICl}$ chez des femmes de en fonction de leur typologie alimentaire entre 13 et 17 ans ; d'après Ananthakrishnan AN et al. (4).
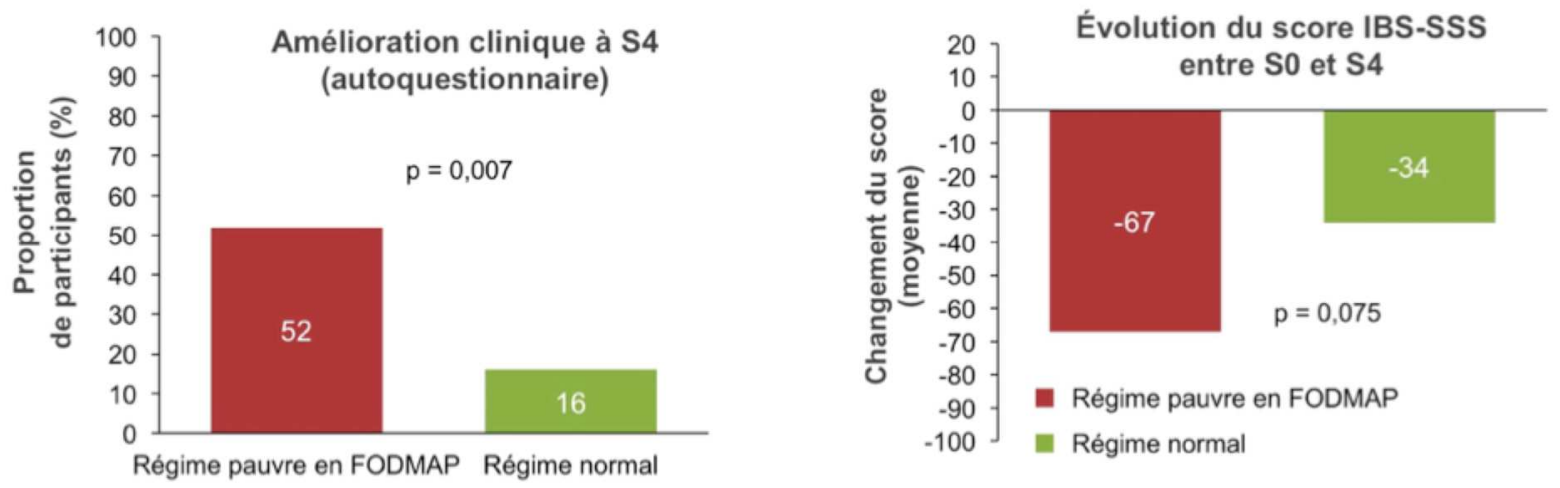

Figure 2: effet d'un régime pauvre en FODMAPs sur les symptômes cliniques de patients atteints de $\mathrm{MICl}$ en rémission qui ont développé un syndrome de l'intestin irritable; d'après Cox SR et al (10). 